\title{
Predicting Shoppers' Interest from Social Interactions Using Sociometric Sensors
}

$\begin{array}{ll}\text { Taemie Kim } & \text { Oliver Brdiczka } \\ \text { MIT Media Lab } & \text { PARC } \\ \text { 20 Ames St } & 3333 \text { Coyote Hill Rd } \\ \text { Cambridge, MA 02139 USA } & \text { Palo Alto, CA 94303 USA } \\ \text { taemie@media.mit.edu } & \text { brdiczka@parc.com } \\ & \\ \text { Maurice Chu } & \text { James "Bo" Begole } \\ \text { PARC } & \text { PARC } \\ \text { 3333 Coyote Hill Rd } & 3333 \text { Coyote Hill Rd } \\ \text { Palo Alto, CA 94303 USA } & \text { Palo Alto, CA 94303 USA } \\ \text { maurice.chu@parc.com } & \text { bo@parc.com }\end{array}$

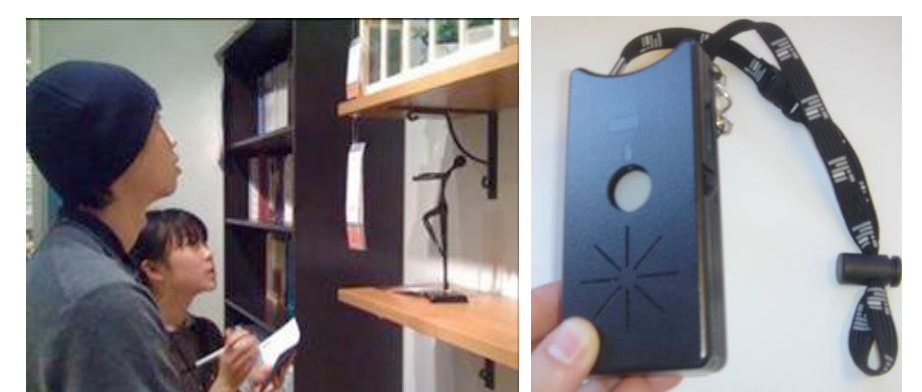

figure 1. We use interaction patterns of couples to predict interest in furniture shopping (left). The couples wear Sociometric badges (right) around their neck allowing the badges to capture their interaction

Copyright is held by the author/owner(s).

CHI 2009, April 4 - 9, 2009, Boston, MA, USA ACM 978-1-60558-246-7/09/04.

\begin{abstract}
Marketing research has longed for better ways to measure consumer behavior. In this paper, we explore using sociometric data to study social behaviors of group shoppers. We hypothesize that the interaction patterns among shoppers will convey their interest level, predicting probability of purchase.

To verify our hypotheses, we observed co-habiting couples shopping for furniture. We have verified that there are sensible differences in customer behavior depending on their interest level. When couples are interested in an item they observe the item for a longer duration of time and have a more balanced speaking style. A real-time prediction model was constructed using a decision tree with a prediction accuracy reaching $79.8 \%$ and a sensitivity of $63 \%$.
\end{abstract}

\section{Keywords}

Shopping, Interest, Behavior modeling, Group

dynamics, Interaction patterns, Sociometric Sensors

\section{ACM Classification Keywords}

H5.3. Group and Organization Interfaces

\section{Introduction}

Retail shopping is more than a trillion dollar business in the U.S. annually [1]. Shopping is often a social experience accompanied by family members or friends. Moreover people have a stronger tendency to shop in 
groups when they are making expensive and important purchases. In this paper we explore whether the verbal and gestural interaction patterns of group shoppers predict their interest levels.

For the scope of this paper, we limited our observations to co-habiting couples shopping for furniture. The decision was based on the syncratic nature of furniture shopping [4], requiring extensive interaction between co-purchasers. Through in-store observations, we collect speech and gestural data of couples and analyze interaction characteristics based on their interest levels. We then use these characteristics to create a prediction model of interest. In summary, our research questions are:

RQ 1. Can we detect significant changes in social behavior when couples are interested in items?

$\boldsymbol{R Q}$ 2. Can we predict interest using sociometric data?

\section{Related Work}

Many researchers have studied social shopping as a collaborative decision making process. Ward [8] investigated decision making procedures for group purchases. Many studied the influence that group shoppers have on each other $[3,7]$. These work state that interaction between co-purchasers strongly influence their purchase decisions.

There have been efforts to use ubiquitous technology to assist shoppers. Begole et al. [2] used computer vision technology to support users in fitting rooms. Kourouthanassis [5] explored using displays on shopping carts to assist shoppers based on their context. These technologies have focused on individual shoppers. Group shopping has been studied in the aa of
e-Commerce but most work have not dealt with realtime or face-to-face collaboration of co-purchasers.

\section{Measuring Couple Dynamics}

To answer our research questions we observed couples shopping for furniture. We recruited 10 female-male couples, age varying from early 20 's to late 40 's. The store chosen was a warehouse style furniture retailer where most of the items are self served by customers. Each participant filled out a questionnaire and answered interview questions before and after shopping. While shopping, participants each wore a Sociometric badge and were followed by an experimenter. They were asked to shop normally with no interaction with the experimenter. Shopping sessions lasted 23 to 85 minutes per couple $(M=55 \min , S D=22 \min , N=10)$.

Sensor Measurements of Social Interactions The Sociometric badge (figure 1, right) is an electronic sensing device that collects and analyzes social behavioral data [6]. Its capabilities include:

- Extracting speech features in real-time: The badge does not record any speech content, but identifies social signals such as speech energy and speaking speed of the wearer. Turn taking or interactivity level is measured through synchronization of multiple badges.

- Measuring body movement using a 3-axis accelerometer: This can detect individual activities such as gesturing, walking, and sitting as well as socia interactions such as body movement mimicry.

- Detecting proximity data using a $2.4 \mathrm{GHz}$ radio or Bluetooth to detect distance of multiple wearers.

- Capturing and identifying two person's face-to-face alignment using an IR sensor: This allows us to detect encounters as well as postural direction. 


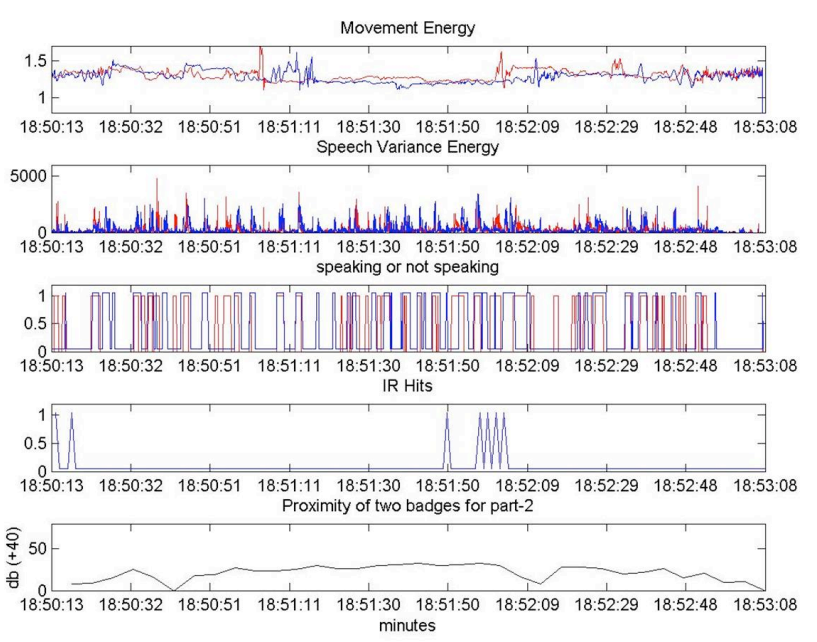

figure 2. Sample data measured by Sociometric badges. The red lines indicate the female's data; and the blue indicates the male's data. The $\mathrm{X}$-axes indicate timeline.

\section{Observing Interest of Shoppers}

While shopping, couples moved about the store observing many items. We say that a couple was engaged with an item when both of the participants were present at the item spending time on discussion or observation. For each item that the couples were engaged with, the experimenter time stamped the start and end time of engagement. Couples were engaged with items for various lengths of time, from a few seconds to a maximum of 455 seconds. However for our analysis, we only considered items with duration of engagement larger than 30 seconds. We collected 99 data points from 10 couples.

The experimenter also took note of actions through which participants explicitly expressed interest. We used four explicit actions as basis to measure interest:
- Purchase: Items that couples ended up purchasing. This shows the highest level of interest.

- Record: Items that couples recorded for further action. This includes taking a picture or writing the item number which was a typical practice in the store.

- Best candidate: Items that couples did not take any action while shopping but answered it to be the best candidate in the post-shopping interview.

- No action: Items that couples were engaged with for more than 30 seconds but did not take any actions and were not stated as best candidate in the postshopping interview.

The number of observations of each action is shown in table 1 . To correlate interest with quantitative measures, we apply a linear scale to these actions also shown in table 1 . For the remainder of the paper, the observed actions will be represented as more interested and less interested according to their linear scale.

\begin{tabular}{|c|c|c|}
\hline Observed Action & $\begin{array}{c}\text { Number of } \\
\text { Observations }\end{array}$ & $\begin{array}{c}\text { Linear Scale of } \\
\text { Interest }\end{array}$ \\
\hline Purchase & 12 & 4 \\
\hline Record & 26 & 3 \\
\hline Best Candidate & 10 & 2 \\
\hline No action & 51 & 1 \\
\hline Total & 99 & \\
\hline
\end{tabular}

table 1. Number of observations of actions that indicate interest level and the corresponding scale of interest.

\section{Characteristics of Interest}

Duration and Interest

Duration of engagement had the strongest correlation with the observed interest. The correlation was positive $(r=0.41, p<.0001)$ indicating that the longer that $a$ 
couple was engaged with an item, the more likely they would show actions that indicate interest. It is interesting that the average duration of interest level 3 and 4 is almost the same, being more than two times the average of interest level 1 (figure 3, top).
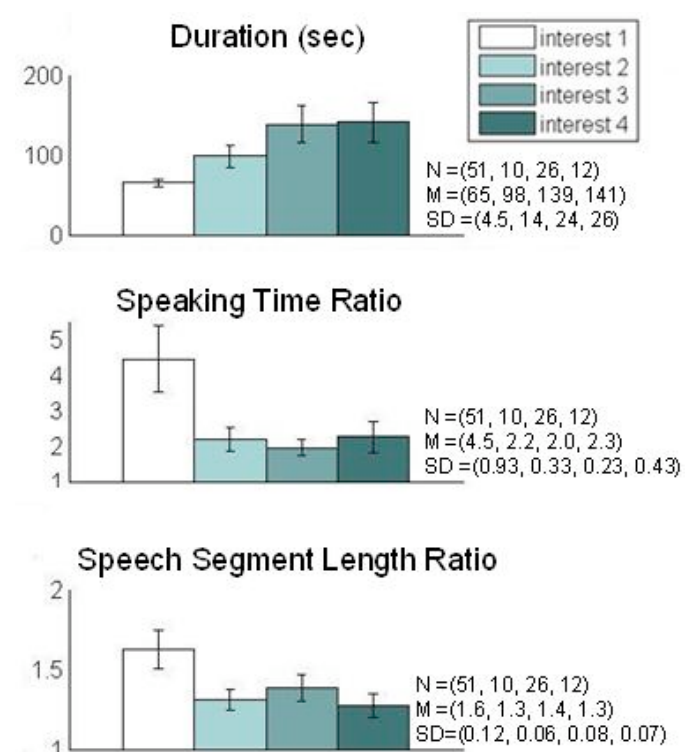

figure 3. (Top) Duration is positively correlated with interest. $\mathrm{r}=0.41, \mathrm{p}<.0001$. (Middle) Speaking time ratio is negatively correlated with interest. $r=-0.23, p<.05$. (Bottom) Average speech segment length ratio is negatively correlated with interest. $r=-0.20, p<.05$

Speaking Time and Interest

Speaking time is defined as the percentage of time that one participant was detected as talking while the other participant was not. We initially investigated if the speaking time of either of the participants had a correlation with interest levels. Looking at the data there was almost no correlation of interest with the speaking time of either of the participants or the average of the two (female: $r=0.05, p=.56$; male: $r=-$ $0.06, p=.63$; average: $r=-0.01, p=.93$ ). This means that there is no consistent difference in speaking time whether or not the couple was interested in an item.

We then looked at the ratio of the two participants' speaking time. If we define the dominant speaker as the person who spoke more and the non-dominant speaker as the person who spoke less, the speaking time ratio is defined as the speaking duration of the dominant speaker divided by the speaking duration of the non-dominant speaker. Hence the minimum value is 1 when both participants spoke an equal amount of time. The ratio of speaking time and interest had a significant negative correlation $(r=-0.23, p<.05)$. The negative correlation indicates that, when a couple is interested in an item they speak for a similar amount of time. As can be seen in figure 3(middle), when couples are not interested in an item the dominant speaker speaks more than four times the non-dominant speaker. In contrast, when couples were interested in an item the dominant speaker speaks only two times the non-dominant speaker. Low speaking time ratio may have mirrored shared enthusiasm in items.

Speech Segment Length and Interest

We define a speech segment as one continuous stream of speech from an individual, regardless of interruption or overlap from the other participant. A segment will end either by an interruption by the other participant that made the speaker stop speaking or a significant length of silence. Speaking time refers to the total amount of speech of an individual, whereas the speech segment length refers to the size of chunks the 
conversation is cut into. Hence, the shorter length of segment length usually corresponds to higher level of interactivity. For each item, average speech segment length was measured for female and male. Speech segment ratio is defined as the longer of the two divided by the shorter of the two. Again, the minimum value is 1 when the average lengths of speech segments are the same for the two participants.

Similar with the correlation of speaking time ratio, a significant correlation was found in the ratio of the two participants' speech segment lengths. The ratio of the average speech segment length is negatively correlated with observed interest $(r=-0.20, p<.05$, figure 3, bottom). This means that as couples were more interested in an item, their average length of speech were more similar. The individual values also had negative correlations but were not statistically significant (female: $r=-0.07, p=.56$; male: $r=-0.18$, $p=.63$; average: $r=-0.12, p=.25$ ). Couples tended to have shorter speech segment lengths when they are interested in an item, but the ratio of the two participants is a stronger indicator of interest.

\section{Prediction of Interest}

Now we investigate the second research question: can we predict interest using just sociometric data? We decided to merge interest level 1 and 2 (taking no action during shopping) and try to distinguish it from level 3 and 4 (took action during shopping). For the rest of this paper, we will denote level 1 and 2 as class "not interested" and level 3 and 4 as class "interested". We used two different machine learning methods to create a prediction model for interest: decision tree and SVM. The base accuracy of the data we collected is $62.2 \%$ which is obtained by predicting that couples are always not interested (sensitivity $=0 \%$ ). We evaluated the predictive models using 10 fold cross-validations.

\section{Decision Tree}

We first used decision trees to construct a descriptive model of interest prediction. The first and most significant attribute to be tested for the decision tree was the duration of engagement. Categorizing all data by a binary test of duration $>141.5 \mathrm{sec}$ raises the prediction rate to $73.7 \%$ (table 2 , left). The ten fold cross-validation accuracy was $72.4 \%(\mathrm{SE}=3.43 \%$ ) being significantly higher than the base accuracy. However, the sensitivity of this model is extremely low correctly predicting only $37 \%$ of the class interested. This is unfortunate since the value of an interest prediction system lies heavily on correctly predicting customers who are interested in an item.

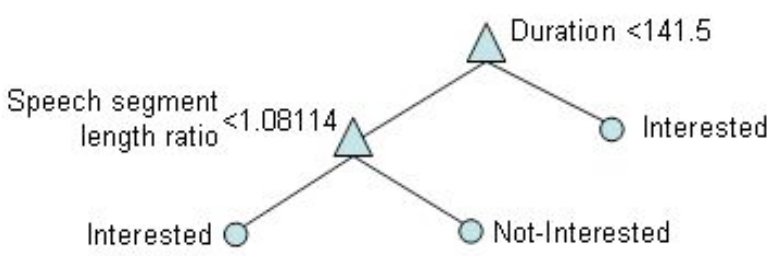

figure 4. Decision tree constructed to predict interest from sociometric data.

By adding another attribute to the decision tree we can further enhance the predictive power as well as the sensitivity. A binary test, speech segment length ratio $<1.08114$, can raise the accuracy rate to $79.8 \%$ (table 2, middle). The ten fold cross-validation accuracy was $77.6 \%$ ( $\mathrm{SE}=3.86 \%$ ), being significantly better than the base accuracy but marginally better than the accuracy achieved by duration only. Sensitivity, on the other hand, increased greatly from $37 \%$ to $63 \%$. 
Support Vector Machines (SVM)

We ran a support vector machine using all attributes we collected with the badge. The attributes included duration, body movement energy, speech energy, speaking time, speech segment length, speech speed, proximity, and number of IR hits. The prediction accuracy rate of SVM model was $76.8 \%$ and the sensitivity was $66 \%$ (table 2 , right).

\begin{tabular}{|c|c|c|c|c|c|c|}
\cline { 2 - 6 } \multicolumn{1}{c|}{} & $\begin{array}{c}\text { Predict } \\
\text { Interested }\end{array}$ & $\begin{array}{c}\text { Predict } \\
\text { Interested }\end{array}$ & $\begin{array}{c}\text { Predict } \\
\text { Interested }\end{array}$ & $\begin{array}{c}\text { Predict } \\
\text { Interested }\end{array}$ & $\begin{array}{c}\text { Predict } \\
\text { Inter } \\
\text { Not }\end{array}$ & $\begin{array}{c}\text { Predict } \\
\text { Intested }\end{array}$ \\
\hline $\begin{array}{c}\text { Actually } \\
\text { Interested }\end{array}$ & 59 & 2 & 55 & 6 & 51 & 10 \\
\hline $\begin{array}{c}\text { Actually } \\
\text { Interested }\end{array}$ & 24 & 14 & 14 & 24 & 13 & 25 \\
\hline
\end{tabular}

table 2. (Left) Decision tree results using only duration, accuracy $=73.7 \%$, sensitivity $=37 \%$. (Middle) Decision tree results using duration and speech segment length ratio, accuracy $=79.8 \%$, sensitivity $=63 \%$. (Right) SVM using all attributes, accuracy $=76.8 \%$, sensitivity $=66 \%$.

The decision tree with both duration and speech segment length ratio achieved the highest prediction accuracy. Although its sensitivity is slightly lower than that of SVM, it has a much lower false positive rate compared ( $10 \%$ and $16 \%$ respectively). This means that there would be less mistakes of approaching customers who are not interested. Furthermore, decision trees can be computed on the badge in realtime, which is not possible for SVM. Hence we suggest using decision trees for future predictions.

\section{Conclusions and Future Work}

We have verified behavioral patterns of couples engaged with items which they are interested in purchasing. They tend to be engaged with the item for a longer period of time and have more balance in their speaking time and lengths of speech segments.

Moreover, we have constructed prediction models of interest having high accuracy in detecting when couples are interested in purchasing an item. We hope to continue this work to develop an automatic customer assistance system which can enhance both the customer and retailer experience.

\section{References}

[1] U.S. Census Bureau. http://www.census.gov

[2] J. Begole, T. Matsumoto, W. Zhang, and J. J. Liu. Responsive mirror: fitting information for fitting rooms. In Proceedings of Workshop on Ambient Persuasion at CHI 2008, Florence, Italy, 2008.

[3] T. L. Childers and A. R. Rao. The influence of familial and peer-based reference groups on consumer decisions. The Journal of Consumer Research, 19(2):51-62, 1992.

[4] H. L. Davis and B. P. Rigaux. Perception of marital roles in decision processes. The Journal of Consumer Research, 1(1):51-62, 1974.

[5] P. Kourouthanassis and G. Roussos. Developing consumer-friendly pervasive retail systems. IEEE Pervasive Computing, 2(2):32-39, 2003.

[6] D. Olguin Olguin, B. Waber, T. Kim, A. Mohan, K. Ara, and A. Pentland. Sensible organizations: Technology and methodology for automatically measuring organizational behavior. IEEE Transactions on Systems, Man, and Cybernetics-B, 2009.

[7] R. Prus. Shopping with companions: Images, influences and interpersonal dilemmas. Qualitative Sociology, 16(2):87-110, 1993.

[8] J. C. Ward and P. H. Reingen. Sociocognitive analysis of group decision making among consumers. The Journal of Consumer Research, 17:245-262, 1990 